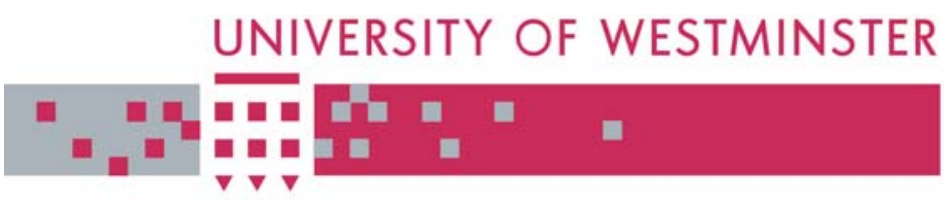

\title{
WestminsterResearch
}

http://www.wmin.ac.uk/westminsterresearch

\section{Design of asymmetrical RF and microwave bandpass filters by computer optimization.}

\author{
Djuradj Budimir \\ George Goussetis \\ School of Informatics
}

Copyright (C) [2003] IEEE. Reprinted from IEEE Transactions on Microwave Theory and Techniques, 51 (4, part 1). pp. 1174-1178.

This material is posted here with permission of the IEEE. Such permission of the IEEE does not in any way imply IEEE endorsement of any of the University of Westminster's products or services. Internal or personal use of this material is permitted. However, permission to reprint/republish this material for advertising or promotional purposes or for creating new collective works for resale or redistribution must be obtained from the IEEE by writing to pubs-permissions@ieee.org. By choosing to view this document, you agree to all provisions of the copyright laws protecting it.

The WestminsterResearch online digital archive at the University of Westminster aims to make the research output of the University available to a wider audience. Copyright and Moral Rights remain with the authors and/or copyright owners.

Users are permitted to download and/or print one copy for non-commercial private study or research. Further distribution and any use of material from within this archive for profit-making enterprises or for commercial gain is strictly forbidden.

Whilst further distribution of specific materials from within this archive is forbidden, you may freely distribute the URL of the University of Westminster Eprints (http://www.wmin.ac.uk/westminsterresearch). 


\title{
Design of Asymmetrical RF and Microwave Bandpass Filters by Computer Optimization
}

\author{
Djuradj Budimir, Senior Member, IEEE, and George Goussetis, Student Member, IEEE
}

\begin{abstract}
This paper presents an optimization-based approach to the design of asymmetrical filter structures having the maximum number of return- or insertion-loss ripples in the passband such as those based upon Chebyshev function prototypes. The proposed approach has the following advantages over the general purpose optimization techniques adopted previously such as: less frequency sampling is required, optimization is carried out with respect to the Chebyshev (or minimax) criterion, the problem of local minima does not arise, and optimization is usually only required for the passband. When implemented around an accurate circuit simulation, the method can be used to include all the effects of discontinuities, junctions, fringing, etc. to reduce the amount of tuning required in the final filter. The design of asymmetrical ridged-waveguide bandpass filters is considered as an example. Measurements on a fabricated filter confirm the accuracy of the design procedure.
\end{abstract}

Index Terms-Bandpass filters, computer optimization, rectangular waveguide, ridged waveguide, waveguide filters.

\section{INTRODUCTION}

A LL-METAL inserts placed in the $E$-plane of a rectangular waveguide along the waveguide axis offer the potential of realizing low-cost, mass-producible, and low dissipation-loss millimeter-wave filters [1]. For the advanced design of such filters, accurate design methods are desirable. General-purpose optimization techniques based on least $p$ th objective functions use general forms of error minimization algorithms [2]. Usually the response of an optimizable filter is sampled at a number of equally spaced frequencies and the error between this sampled response and the desired response is computed at each frequency. The optimization program, through an iterative process, reduces this error to a minimum, arriving at a final filter design in terms of the optimized filter parameters. These optimization techniques cannot be guaranteed to satisfy filter specifications and may even converge to a local minimum. This paper attempts to show how problems within the scope of asymmetrical microwave filter design may be formulated effectively as optimization problems, to explain the differences between these formulations, and to indicate an appropriate optimization method that can be implemented in situations when the classical synthesis approach is inappropriate. When a common approach to the design of microwave filters results in a design passband, which differs considerably from that which is specified, optimization

Manuscript received March 14, 2002; revised November 6, 2002. This work was supported by the Engineering and Physical Sciences Research Council, U.K., under Grant GR/K58634.

The authors are with the Wireless Communications Research Group, Department of Electronic Systems, University of Westminster, London W1W 6UW, U.K. (e-mail: budimid@wmin.ac.uk).

Digital Object Identifier 10.1109/TMTT.2003.809623 is required to tune the filter dimensions to achieve a design that meets certain requirements.

An optimization procedure to optimize symmetrical bandpass filters [3] has already proven its suitability. The procedure is extended (modified) here to optimize asymmetrical RF and microwave bandpass filters. This method searches for tuning points in the filter transfer function and forces the minimums, as well as the maximums (peaks), of the ripple levels at these points to have specified values. If an $n$ th-degree filter is present, there are $n-1$ maximums, $n$ minimums, and two band edges making $2 n+1$ optimization parameters. The method requires knowledge of the filter insertion or return loss at these points. The method will generate a set of equations that are solved to give a new set of parameter values. The cycle is then repeated until the filter characteristic is within an arbitrarily close value to the desired specification. This technique optimizes the passband of a filter with respect to the Chebyshev (or minimax) criterion [5].

Formulation of the equal-ripple optimization in the context of the design of asymmetrical microwave bandpass filters, in terms of insertion loss is given in Section II. The numerical implementation of equal-ripple optimization, in the context of the design of an asymmetrical ridged waveguide bandpass filter, is presented in Section III. The design example is presented in Section IV. Measurements on a fabricated filter confirm the accuracy of the design procedure.

\section{DESCRIPTION OF THE ALGORITHM}

Assume that an $n$ th-degree asymmetrical ridged-waveguide bandpass filter (Fig. 1) has an insertion-loss response $L_{I}$ of the form shown in Fig. 2. It exhibits $(m-1) / 2(m=2 n+1)$ nonzero minima, the minima of which occur at the frequencies $f_{2}, f_{4}, \ldots, f_{m-1}$. There are $(m-3) / 2$ ripples, the maxima of which occur at the frequencies $f_{3}, f_{5}, \ldots, f_{m-2}$. All of these $m-2$ frequencies lie within the specified passband $f_{l} \Rightarrow f_{u}$.

The deviation of a ripple maximum from the maximum allowed insertion loss in the passband $L_{I r}$ is a function of the $m=2 n+1$ values required to specify the bandpass filter. There are $2 n-1$ such functions

$$
\begin{aligned}
& E_{i}=L_{I}\left(f_{i}\right)-L_{I r}, \quad i=3,5, \ldots, 2 n-1 \\
& E_{i}=L_{I}\left(f_{i}\right), \quad i=2,4, \ldots, 2 n
\end{aligned}
$$

which have to be zero in order to satisfy the filter specification. $E_{1}$ and $E_{m}$ are defined by

$$
\begin{aligned}
E_{1} & =L_{I}\left(f_{l}\right)-L_{I r} \\
E_{m} & =L_{I}\left(f_{u}\right)-L_{I r} .
\end{aligned}
$$



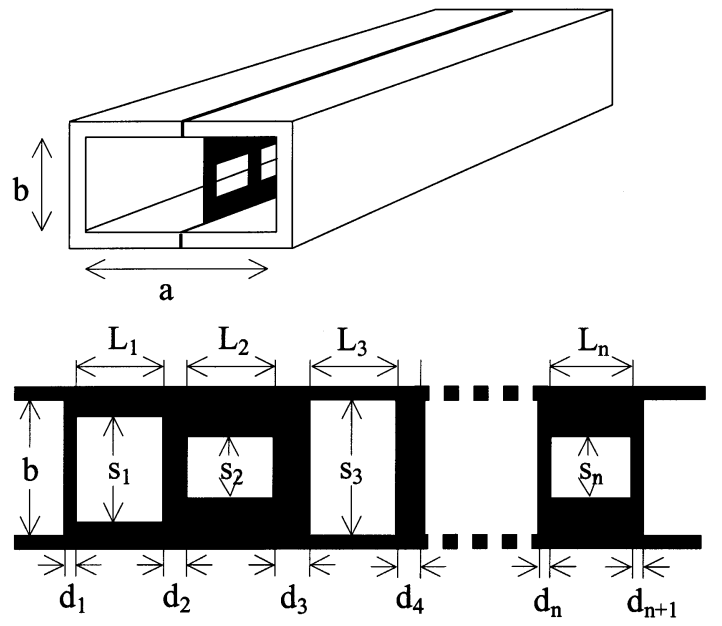

Fig. 1. Configuration of the ridged-waveguide filter structure.

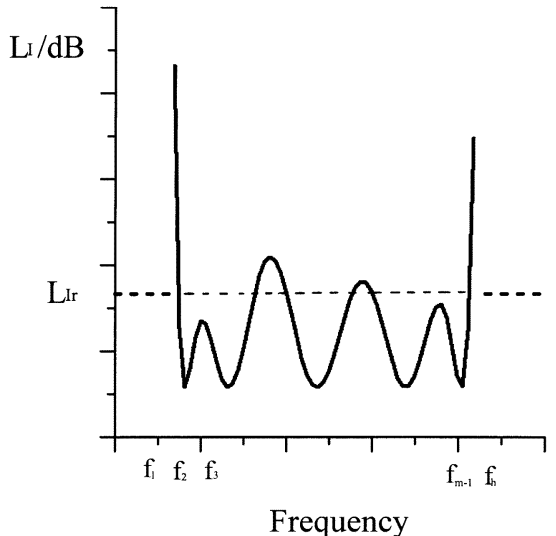

Fig. 2. Scheme for the numerical optimization of an asymmetrical filter.

$E_{1}$ and $E_{m}$ are also functions of the $m=2 n+1$ parameter values of the asymmetrical filter.

The specification

$$
\begin{aligned}
& L_{I}\left(f_{i}\right) \leq L_{I r} \quad f_{l} \leq f_{i} \leq f_{u}, \quad i=1,3,5, \ldots, 2 n-1 \\
& L_{I}\left(f_{i}\right)=0 \quad f_{l} \leq f_{i} \leq f_{u}, \quad i=2,4, \ldots, 2 n
\end{aligned}
$$

is satisfied when

$$
E_{i}=0, \quad i=1,2,3 \ldots, m .
$$

This is a system of $m=2 n+1$ nonlinear equations in $m=2 n+1$ variables, which, in practice, needs to be solved iteratively. The parameter values of a filter satisfying (4) and (5) can be obtained by solving (6). The $E_{i}(i=1, \ldots, m)$ can be regarded as the components of an $m$ dimensional error vector. By equating each of these components to zero (a vector process) rather than minimizing the magnitude of the vector (a scalar process), optimization is carried out. To apply an iterative nonlinear equation solver, it is necessary for a given set of filter parameter values to know the insertion loss only at the band-edge frequencies and at the ripple maxima and minima. However, the frequencies at which the ripple maxima and minima occur are unknown and are functions of the filter parameter values. For a

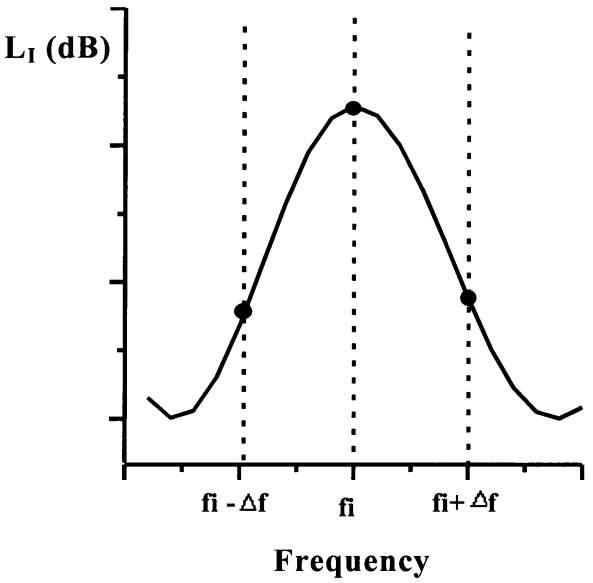

(a)

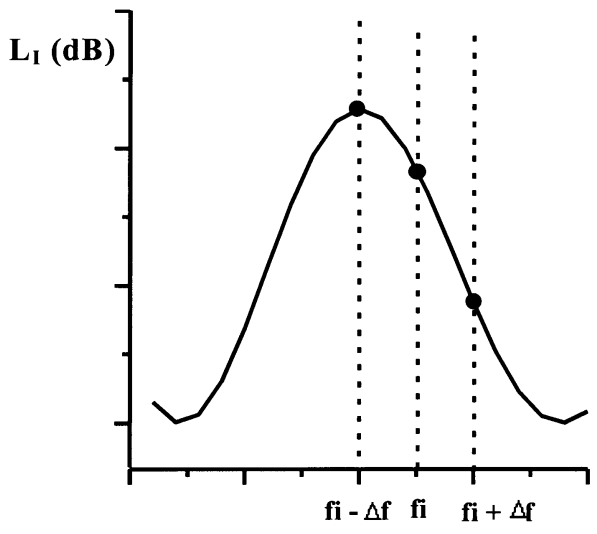

Frequency

(b)

Fig. 3. (a) $f_{i}$ on maximum and (b) off maximum.

given set of filter parameter values, these frequencies can be approximately located by calculating the insertion loss on a coarse sample of frequency points in the passband. The ripple maxima and minima are shown in Figs. 3 and 4, respectively.

Figs. 3(a) and 4(a) show $f_{i}$ correctly centred at the maximum and minimum. In Figs. 3(b) and 4(b), the sample frequency $f_{i}$ is a little off so the function is sampled at frequencies slightly higher and lower. By finding an equivalent parabola

$$
L_{I}=a+b f+c f^{2}
$$

passing through the three points at $f_{i}-\Delta f, f_{i}$ and $f_{i}+\Delta f$, a correction is derived, which can be applied to the frequency $f_{i}$ to bring it closer to the extreme (minima or maxima). By using quadratic interpolation [2] in the last few iterations, the correct location and amplitude of the ripple maxima and minima can be found.

The necessary condition for the maximum of $L_{I}(f)$ is that

$$
\frac{d L_{I}}{d f}=b+2 c f=0
$$

i.e.,

$$
f=-\frac{b}{2 a}
$$




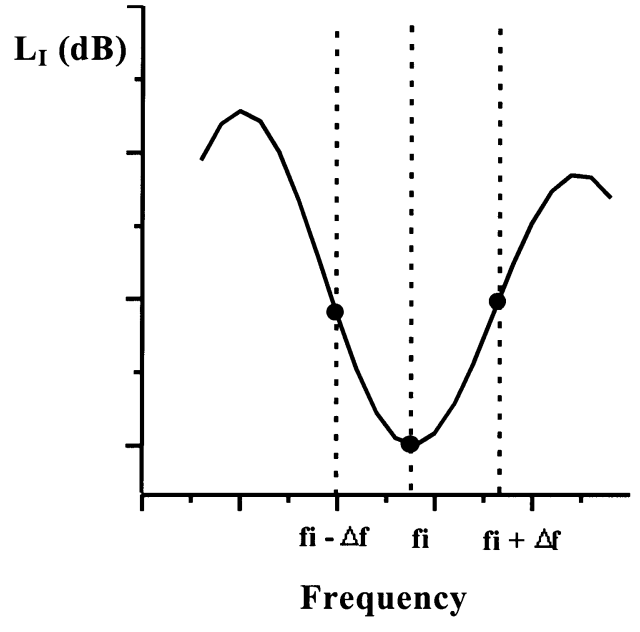

(a)

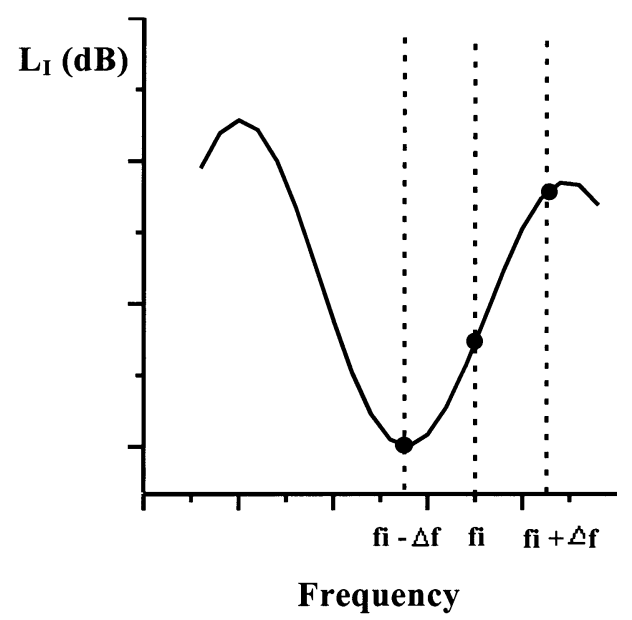

(b)

Fig. 4. (a) $f_{i}$ on minimum and (b) off minimum.

where $f$ locates the maximum of $L_{I}(f)$. The sufficiency condition for the maximum of $L_{I}(f)$ is that

$$
\frac{d^{2} L_{I}}{d f^{2}}<0
$$

The correct location and amplitude of the ripple maxima can be found by using the above procedure (quadratic interpolation) in the last few iterations.

\section{Algorithm For SOlving THE SYStem OF NONLINEAR EQUATIONS}

The Newton-Raphson method [3] is a rapidly convergent technique for the solution of a system of nonlinear equations if a good initial approximation is available. The number of times the function is evaluated in the process of finding its root is the usual measure of computational effort. This includes function evaluations required to calculate derivatives numerically. By using finite difference, the Jacobian matrix of the $2 n+1$ nonlinear functions defined by (1)-(6) can be calculated numerically. For a given set of filter parameters, the finite-difference calculation of the Jacobian matrix requires the evaluation of

$$
\begin{aligned}
& E_{i}\left(x_{1}, x_{2}, \ldots, x_{j}+\delta x_{j}, \ldots, x_{2 n+1}\right), \\
& \quad i=1,2, \ldots, 2 n+1 ; \quad j=1,2, \ldots, 2 n+1
\end{aligned}
$$

where $x_{1}, x_{2}, \ldots, x_{2 n+1}$ denote the $2 n+1$ dimensions required to specify a asymmetrical ridged waveguide filter (see Fig. 1).

For odd $n$

$$
\begin{aligned}
& x_{j}=d_{j}, \quad j=1,2, \ldots, \frac{2 n+2}{2} \\
& x_{j}=l_{j-(2 n+2 / 2)}, \quad j=\frac{n+3}{2}, \frac{n+5}{2}, \ldots, 2 n+1 .
\end{aligned}
$$

For even $n$

$$
\begin{aligned}
& x_{j}=d_{j}, \quad j=1,2, \ldots, \frac{2 n}{2}+1 \\
& x_{j}=l_{j-1-2 n / 2}, \quad j=\frac{2 n}{2}+2, \frac{2 n}{2}+3, \ldots, 2 n+1 .
\end{aligned}
$$

Denoting by $x$ and $E$ the $2 n+1$ dimensional vectors with components $x_{i}(i=1,2, \ldots, 2 n+1)$ and $E_{i}(i=1, \ldots, 2 n+1)$, the Newton-Raphson method has the general form [6]

$$
X^{k}=X^{k-1}-J^{-1}\left(x^{k-1}\right) E\left(x^{k-1}\right)
$$

where $k$ is the iteration number $(k=1,2, \ldots)$ and $J^{-1}$ is the inverse of the $m \times m$ Jacobian matrix evaluated at $x^{k-1}$. The response and errors after each iteration are computed again with the new corrected parameters until the errors are judged to be sufficiently small.

The Jacobian matrix of the $m=2 n+1$ nonlinear functions is defined by

$$
\begin{aligned}
J_{i j}= & {\left[\begin{array}{cccc}
\frac{\partial E_{1}}{\partial x_{1}} & \frac{\partial E_{1}}{\partial x_{2}} & \ldots & \frac{\partial E_{1}}{\partial x_{m}} \\
\frac{\partial E_{m}}{\partial x_{1}} & \frac{\partial \dot{E}_{m}}{\partial x_{2}} & \ldots & \frac{\partial E_{m}}{\partial x_{m}}
\end{array}\right] \ldots, } \\
i=1,2, \ldots, 2 n+1 ; & j=1,2, \ldots, 2 n+1 .
\end{aligned}
$$

All blocks defined by (15) can be calculated numerically using finite difference for a given set of filter parameter values as

$$
\begin{aligned}
\frac{\partial E_{i}}{\partial x_{j}} \cong \frac{E i\left(x_{j}+\delta x_{j}\right)-E_{i}\left(x_{j}\right)}{\delta x_{j}} \ldots, & \\
& i=1,2, \ldots, 2 n+1 ; \quad j=1,2, \ldots, 2 n+1 .
\end{aligned}
$$

The choice of $\delta x_{j}$ was arbitrary and was taken to be roughly a one-thousandth part of $x_{j}$.

\section{NUMERICAL IMPLEMENTATION OF EQUAL-RIPPLE OPTIMIZATION}

To apply the equal-ripple optimization technique described in Section II to the design of asymmetrical bandpass filters, it is necessary, for a given set of insert dimensions, to be able to calculate the insertion loss on a sample of frequency points within the specified passband. For an asymmetrical ridged-waveguide bandpass filter, the insertion loss can be expressed in terms of 
an $A B C D$ matrix [4]. The matrix representation of the whole filter is

$$
\begin{aligned}
\left(\begin{array}{cc}
A & j B \\
j C & D
\end{array}\right)= & \left(\begin{array}{cc}
A_{1} & j B_{1} \\
j C_{1} & D_{1}
\end{array}\right) \cdot\left(\begin{array}{cc}
A_{\text {res }}^{1} & j B_{\text {res }}^{1} \\
j C_{\text {res }}^{1} & D_{\text {res }}^{1}
\end{array}\right) \\
& \times\left(\begin{array}{cc}
A_{2} & j B_{2} \\
j C_{2} & D_{2}
\end{array}\right) \cdots\left(\begin{array}{cc}
A_{\text {res }}^{2 n} & j B_{\text {res }}^{2 n} \\
j C_{\text {res }}^{2 n} & D_{\text {res }}^{2 n}
\end{array}\right) \\
& \times\left(\begin{array}{cc}
A_{2 n+1} & j B_{2 n+1} \\
j C_{2 n+1} & D_{2 n+1}
\end{array}\right)
\end{aligned}
$$

where

$$
\begin{aligned}
& \left(\begin{array}{cc}
A_{\mathrm{res}}^{i} & j B_{\mathrm{res}}^{i} \\
j C_{\mathrm{res}}^{i} & D_{\mathrm{res}}^{i}
\end{array}\right) \\
& \quad\left(\begin{array}{cc}
\cos \theta_{i} & j \frac{f}{\sqrt{f^{2}-f_{c i}^{2}}} \sin \theta_{i} \\
j \frac{\sqrt{f^{2}-f_{c i}^{2}}}{f} \sin \theta_{i} & \cos \theta_{i}
\end{array}\right), \ldots, \\
& \quad i=1,2,3, \ldots, 2 n
\end{aligned}
$$

with

$$
\theta_{i}=\frac{2 \pi L_{i}}{\lambda_{g i}}
$$

in which $L_{i}$ is the ridged-waveguide resonator length, $\lambda_{g i}$ is the wavelength in the ridged-waveguide resonator for each frequency, and $f_{c}$ is the cutoff frequency in the ridged-waveguide resonator. The overall filter response [insertion loss $\left(L_{I}\right)$ ] can be expressed in terms of elements of the total $A B C D$ matrix of the filter at each frequency (by directly combining the $A B C D$ matrices of the individual filter sections) as

$$
L_{I}=20 \log _{10}\left(\frac{A+B+C+D}{2}\right) .
$$

The elements of the $A B C D$ matrices of the individual filter sections are calculated using the mode-matching method [8]-[11]. The propagation constants of the eigenmodes in ridged waveguides are related to the cutoff frequencies. The transcendental equation of the eigenvalue of the $n$th mode in the ridged waveguide was solved numerically. However, due to the singular behavior of the magnetic field at the edges of the septa, a large number of modes need to be included in the field expansions to ensure good convergence. This is similar to the situation for the septum in the rectangular waveguide, and is due to the singular behavior of the magnetic field at the edges of the septum.

Neither accurate numerically fitted closed-form expressions, nor accurate design tables for the electrical parameters of the $E$-plane septa in terms of septum dimensions (length and thickness) and frequency are yet available. Thus, the accurate design of ridged-waveguide filters requires the direct calculation of the electrical parameters of the $E$-plane septa. This highlights the need in the optimized design of these filters for optimization techniques, which minimize the number of calculations of the electrical parameters of the $E$-plane septa. A good approximate design of ridged-waveguide filters can be obtained by the procedure described in [4]. This procedure tries to implicitly include the actual frequency dependence of the $E$-plane septa and results in passbands that nearly meet design specifications such as the two passband edge frequencies yielding $\lambda_{g 1}$ and $\lambda_{g 2}$, passband return loss $\left(L_{R}\right)$, stopband attenuation $\left(L_{I}\right)$,

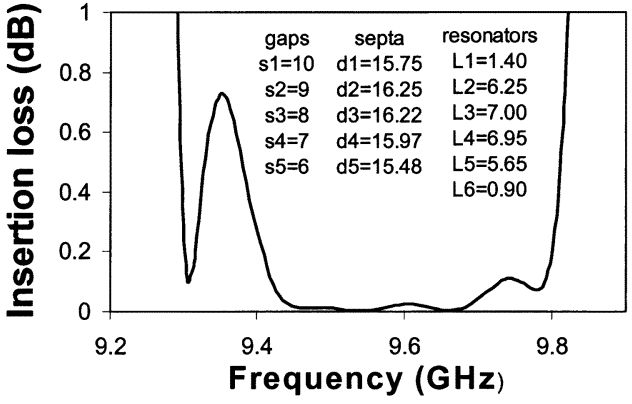

Fig. 5. Calculated insertion loss before optimization.

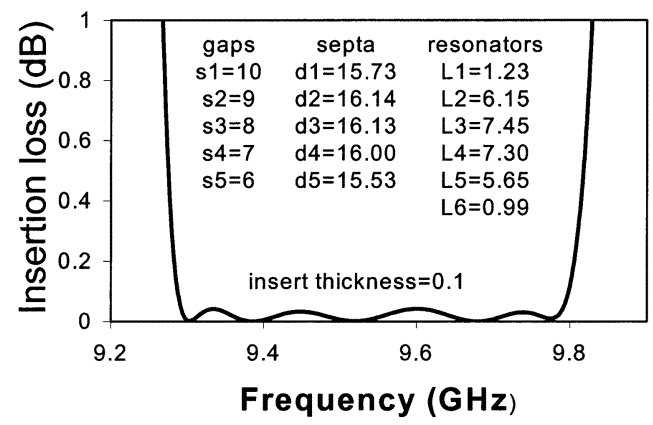

Fig. 6. Calculated insertion loss after optimization.

the waveguide housing dimensions $(a, b)$, the metal septum thickness $(t)$, and the ridged-waveguide gap $(s)$. It is, therefore, adopted in this paper as a means of generating a starting point for optimization.

\section{NUMERICAL AND EXPERIMENTAL RESULTS}

In order to illustrate the new approach, a five-resonator $X$-band ridged-waveguide bandpass filter in WG-16, in which the widths of the ridges are arbitrarily chosen (see Fig. 1), with passband specification $L_{I}(f) \leq 0.05 \mathrm{~dB}, 9.30 \mathrm{GHz}$ $\leq f \leq 9.80 \mathrm{GHz}$ has been designed. Fig. 5 shows the calculated passband insertion loss of the ridged-waveguide filter (ridged-waveguide gap $\left(s_{1}\right)=10.00 \mathrm{~mm}$, ridged-waveguide gap $\left(s_{2}\right)=9.00 \mathrm{~mm}$, ridged-waveguide gap $\left(s_{3}\right)=8.00 \mathrm{~mm}$, ridged-waveguide gap $\left(s_{4}\right)=7.00 \mathrm{~mm}$ and ridged-waveguide gap $\left(s_{5}\right)=6.00 \mathrm{~mm}$, respectively) using the approximate method described in [8].

This approximate design was used as a starting point for equal-ripple optimization. The passband insertion loss calculated using the insert dimensions obtained on convergence is shown in Fig. 6. The insert dimensions are shown in this figure. The mode-matching method with $35 \mathrm{TE}$ and $20 \mathrm{TM}$ modes is used throughout the optimization. The photograph of a five-resonator asymmetrical ridged-waveguide bandpass filter with the corresponding waveguide housing is shown in Fig. 7. The calculated insertion loss and return loss of the final design are shown in Fig. 8. The measured insertion loss and return loss of the fabricated design are also included in this figure. The filter was fabricated using a copper metal insert, which was realized using a drill plotter (LPKF Protomat 60). The measured insertion loss in the passband was less than $0.50 \mathrm{~dB}$. The simulated and experimental responses are in very good 


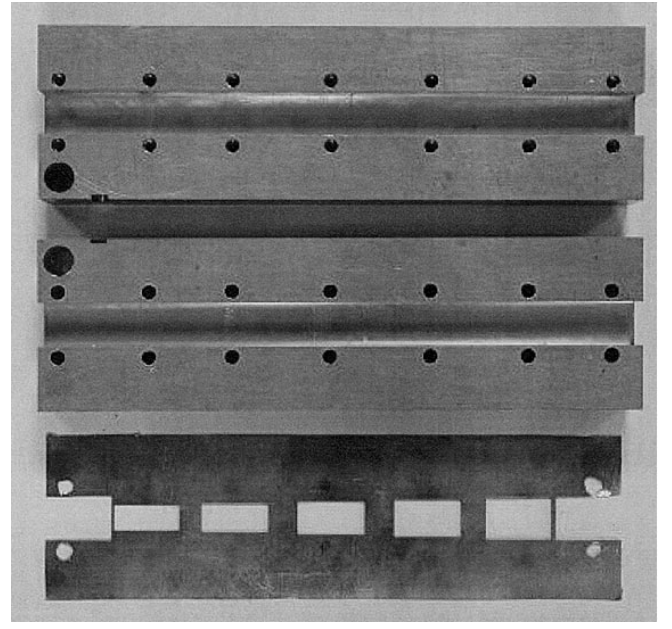

Fig. 7. Photograph of fabricated prototype.

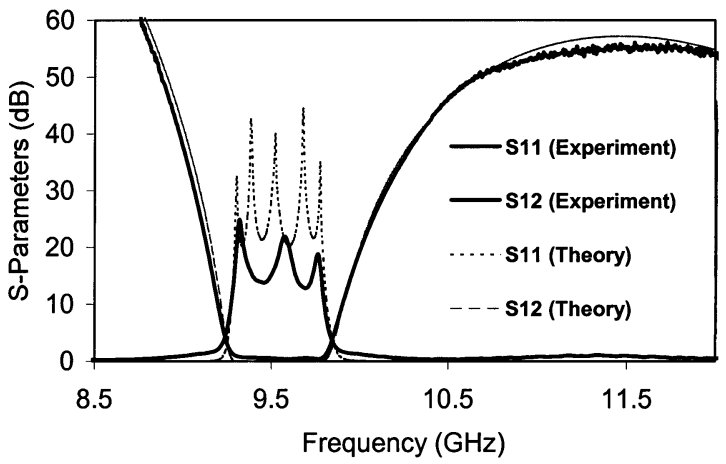

Fig. 8. Theoretical and experimental results of fabricated prototype (dimensions as in Fig. 6).

agreement. Any disagreement between theory and experiment are due to the limited tolerances of the fabrication method.

\section{CONCLUSIONS}

An optimization-based procedure for the accurate design of asymmetrical RF and microwave filters has been presented. This is a simple, fast, and reliable optimization method for the final optimization of asymmetrical RF and microwave filter structures having the maximum number of return- or insertion-loss ripples in the passband such as those based upon Chebyshev function prototypes. Given an accurate simulation of the structure, the method will handle bandpass, low-pass, and high-pass types, and will allow the return- or insertion-loss characteristic to be nonequiripple. Its validity has been demonstrated for the case of a nonuniform waveguide $E$-plane filter. The developed method has also predicted the stopband performance and has provided a guiding tool for the design of filters with strict stopband specifications. The electromagnetic analysis of the discontinuities in ridged-waveguide filters has been performed using a mode-matching method. Experimental evidence demonstrates the validity of the design procedure.

\section{REFERENCES}

[1] F. Arndt, "The status of rigorous design of millimeter wave low insertion loss fin-line and metallic E-plane filters," J. Inst. Electron. Telecommun. Eng., vol. 34, no. 2, pp. 107-119, 1988.
[2] K. C. Gupta, R. Garg, and R. Chadha, CAD of Microwave Circuits. Dedham, MA: Artech House, 1981.

[3] D. Budimir, "Optimized E-plane bandpass filters with improved stop band performance," IEEE Trans. Microwave Theory Tech., vol. MTT-45, pp. 212-220, Feb. 1997.

[4] — Generalized Filter Design by Computer Optimization: Artech House, 1998.

[5] M. Hasler and J. Neiryuck, Electrical Filters. Dedham, MA: Artech House, 1986.

[6] J. M. Ortega and W. C. Rheinboldt, Iterative Solution of Nonlinear Equations in Several Variables. New York: Academic, 1970.

[7] R. Levy, "A generalized design technique for practical distributed reciprocal ladder networks," IEEE Trans. Microwave Theory Tech., vol. MTT-21, pp. 519-526, Aug. 1973.

[8] Y. C. Shih, "The mode-matching method," in Numerical Techniques for Microwave and Millimeter-Wave Passive Structures, T. Itoh, Ed. New York: Wiley, 1989, pp. 592-621.

[9] J. P. Montgomery, "On complete eigenvalue solution of ridged waveguide," IEEE Trans. Microwave Theory Tech., vol. MTT-19, pp. 547-555, June 1971.

[10] G. Goussetis and D. Budimir, "Waveguide filters with improved stopband performance," presented at the 30th Eur. Microwave Conf., Paris, France, Oct. 2-6, 2000.

[11] — "Bandpass filters with improved stopband performance," presented at the Asia-Pacific Microwave Conf., Sydney, Australia, Dec. 3-6, 2000.

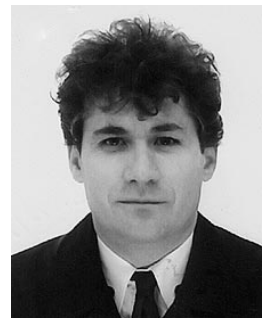

Djuradj Budimir (S'89-M'92-SM'02) was born in Serbian Krajina (formerly Yugoslavia). He received the Dipl. Ing. and M.Sc. degrees in electronic engineering from the University of Belgrade, Belgrade, Serbia, respectively, and the Ph.D. degree in electronic and electrical engineering from The University of Leeds, Leeds, U.K.

In March 1994, he joined the Department of Electronic and Electrical Engineering, Kings College London, University of London, London, U.K., as a Post-Doctoral Research Fellow. Since January 1997, he has been with the Department of Electronic Systems, University of Westminster, London, U.K. He owns DBFILTER, a software and consulting company. He has authored or coauthored over 100 technical papers in the fields of RF, microwave, and millimeter-wave computer-aided design (CAD). He also authored Generalized Filter Design by Computer Optimization (Norwood, MA: Artech House, 1998) and the software and users manual EPFIL-Waveguide E-Plane Filter Design (Norwood, MA; Artech House, 2000). His research interests include analysis and design of hybrid and monolithic microwave integrated circuits such as amplifiers and filters, dielectric-resonator filters for modern communications systems, the application of numerical methods to the electromagnetic-field analysis of passive microwave and millimeter-wave circuits, and the design of waveguide filters and multiplexing networks for microwave and millimeter-wave applications. He has served on the Editorial or Review Boards of the IEE Electronic Letters, and the Proceedings of the IEE-Microwaves, Antennas, and Propagation.

Dr. Budimir is a Chartered Electrical Engineer (CEng). He is a member of the Institution of Electrical Engineering (IEE), U.K. He has served on the Editorial or Review Boards of various technical journals, including the IEEE MiCROWAVE AND WIRELESS COMPONENTS LETTERS, the IEEE TRANSACTIONS ON MicrowaVe TheOry and TeChNiques, and the IEEE TRANSACTIONS ON CiRCUITS AND Systems. He has also served on the Technical Program Committee for the TELSIKS $(1997,1999,2001)$.

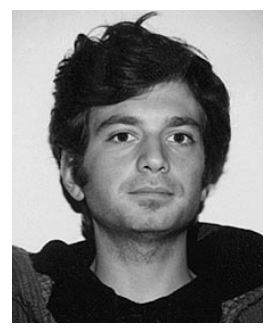

George Goussetis ( $\mathrm{S}^{\prime} \mathrm{O} 2$ ) was born in Athens, Greece, in 1976. He received the Electrical and Computer Engineering degree from the National Technical University of Athens, Athens, Greece, in 1998, and the Ph.D. degree in the area of waveguide filters from the University of Westminster, London, U.K., in 2002.

In 1999, he joined the Wireless Communications Research Group, University of Westminster, as a Research Assistant. His research interests include the design of microwave passive components and the application of numerical methods to electromagnetic-field analysis. 